\title{
Oral preload of calcium reduces food intake via enhanced PYY secretion in rats
}

\section{Abstract \\ Introduction}

Dietary calcium has been proposed to reduce appetite (or to enhance satiety) in human studies. However, underlying mechanisms are still unclear. In animal and cell studies, it has been demonstrated that activation of the calcium-sensing receptor induced secretion of anorexic gut hormones such as cholecystokinin (CCK) and glucagon-like peptide-1 (GLP-1) from enteroendocrine cells. In the present study, we tested the hypothesis that calcium suppresses appetite thorough enhanced gut hormone secretion, by using rats.

\section{Materials and Methods}

Male Sprague Dawley rats were maintained by feeding a standard diet (AIN-93G, $n=6-8$ per group). As calcium sources, calcium chloride, calcium carbonate, and calcium lactate were tested. These calcium salts were orally preloaded in fasted rats by using a feeding tube, and subsequent food intake was monitored until 24 hours. To assess conditioned taste aversion, saccharin preference test was conducted after conditioning with calcium or lithium chloride. To investigate involvements of gut hormones such as CCK, GLP-1, and peptide-YY (PYY), specific receptor antagonists for respective gut hormones were intraperitoneally injected just after oral preload of calcium, and then food intake was monitored. Portal blood samples were collected 15 or 30 min after oral preload of calcium for measurement of gut hormones by ELISA.

\section{Results and discussion}

At the same dose of calcium $(150 \mathrm{mg} / \mathrm{kg})$, preload of calcium chloride reduced food intake for 4 hours compared to preload of the control solution $(\mathrm{P}<0.05)$, while other compounds had minor effects on food intake. Saccharin preference ratio was only reduced by conditioning with lithium chloride $(\mathrm{P}<0.01)$, but not by that with calcium compounds, indicating no conditional taste aversion was occurred by calcium. Suppressive effect of calcium chloride on food intake was partially reversed by pretreatment with a PYY receptor antagonist (BIIE0246) but not by that with a CCK- or a GLP-1 receptor antagonist. Portal PYY concentrations were higher in calcium chloride-treated rats than in the control rats $(\mathrm{P}<0.05), 15 \mathrm{~min}$ after the preload and re-feeding. Changes in serum calcium concentrations were not observed by preload of calcium.

These results suggest that oral preload of calcium chloride reduces subsequent food intake via enhanced PYY secretion in rats.

\section{Conflict of Interest}

There is no conflict of interest. 